\title{
Apoptotic-like death occurs through a caspase-independent route in colon carcinoma cells undergoing mitotic catastrophe
}

Laia Llovera, Sylvia Mansilla and José Portugal*

Instituto de Biologia Molecular de Barcelona, CSIC, Parc Cientific de Barcelona, Barcelona, Spain

* Corresponding author:

José Portugal

Instituto de Biologia Molecular de Barcelona, CSIC,

Parc Cientific de Barcelona,

Baldiri Reixac, 10

E-08028 Barcelona, Spain

Tel: +34-93- 4034959

FAX: +34-93- 4034979

E-mail: jpmbmc@ibmb.csic.es 


\begin{abstract}
We have examined the relationship between chemotherapy-induced mitotic catastrophe and cell death by apoptosis in both wild-type and $p 53^{(--)}$HCT116 human colon carcinoma cells treated with nanomolar concentrations of paclitaxel (PTX), a drug that acts on tubulin altering the normal development of mitosis. After treatment, HCT116 cells entered mitosis regardless of the presence of functional $\mathrm{p} 53$, which resulted in changes in the distribution of cells in the different phases of the cell cycle, and in cell death. In the presence of PTX, the percentage of polyploid cells observed was higher in p53-deficient cells, indicating that mitotic slippage was favored compared to wild-type cells, with the presence of large multinucleate cells. PTX caused mitotic catastrophe and about $50-60 \%$ cells that were entering an aberrant mitosis died through an apoptotic-like pathway characterized by the presence of phosphatidylserine in the outer cell membrane, which occurred in the absence of significant activation of caspases. Lack of p53 facilitated endoreduplication and polyploidy in PTX-treated cells, but cells were still killed with similar efficacy through the same apoptotic-like mechanism in the absence of caspase activity.
\end{abstract}

Keywords: Apoptosis; Caspases; HCT116 cells; Mitotic catastrophe; Paclitaxel

\title{
1. Introduction
}

Mitotic catastrophe is a term used to describe cell death that occurs during or after a faulty mitosis. Mitotic catastrophe is the principal form of cell death induced by ionizing radiation and it has been identified as the main response to several antitumor drugs [1-3]. The rising of mitotic catastrophe appears to be controlled by a variety of cell-cycle-specific proteins (such as the cyclin B1-dependent kinase Cdk1, polo-like kinases and Aurora kinases), cell-cycle checkpoint proteins, survivin, p53, and members of the Bcl-2 family [3,4].

Moderate DNA damage activates p53, and wild-type p53 appears to promote two antiproliferative responses: apoptosis and senescence, whereas it can inhibit mitotic catastrophe [1]. Because tumor cells are frequently deficient in factors controlling the cell-cycle checkpoints, particularly functional p53, they may be predisposed to mitotic catastrophe after chemotherapy $[1,5,6]$. DNA damage combined with G2/M checkpoint deficiency leads cells to multinucleation, which may be followed by cell death $[1,3,7]$. Apoptosis and necrosis have been observed following multinucleation [8-11], and it has been proposed that mitotic catastrophe might not constitute a "genuine" cell death mechanism, but an oncosuppressive pathway that can precede cell death $[12,13]$. 
Although apoptosis can follow a faulty mitosis, mitotic catastrophe has been regarded as fundamentally different from apoptosis $[1,14]$ mainly because the overexpression of several antiapoptotic genes enhances the frequency of defective mitosis $[1,15]$. This has raised the question of whether the large multinucleate cells that emerge after a faulty mitosis may become more invasive. Several in vivo and in vitro models have shown that the different cell death pathways are somewhat associated, and mitotic catastrophe can be accompanied by the release of pro-apoptotic proteins and caspase activation, which would entail that mitotic catastrophe shares some events used to define apoptosis $[4,16]$. Thus, mitotic catastrophe may end in apoptosis-like cell death when caspases are active or in a caspase-independent cell death, and cells may also be committed to undergoing necrosis $[3,4,17]$. However, further details about what triggers the different responses are needed in the pursuit of targeting mitotic catastrophe as an antitumor strategy.

Paclitaxel (PTX), also known as Taxol, is a plant alkaloid commonly used in the treatment of several human carcinomas $[5,18]$. It binds with high affinity to the $\beta$-subunit of tubulin causing decreased dynamic instability, increased microtubule rigidity and mitotic arrest, yet PTX-treated cells can show endoreduplication. The cellular effects of this drug depend on dose and treatment schedule $[19,20]$, as well as on the p53 protein levels [21]. In the nanomolar range, it induces sustained mitotic arrest [18] and can trigger apoptosis [19,22]. In general, cells that fail to complete cytokinesis after mitotic arrest caused by DNA or spindle damage become multinucleated and undergo mitotic catastrophe [2,23], which eventually ends in cell death or senescence.

We sought to gain new insights into the effect of nanomolar PTX concentrations on the final fate of cells undergoing mitotic catastrophe, as well as to examine whether apoptosis and necrosis are involved in the final fate of cells dying by mitotic catastrophe, during either mitosis or following re-entry in cell cycle after mitotic slippage. To this end, we have used HCT116 colon carcinoma cells bearing wild-type $p 53$ and HCT116 cells whose $p 53$ had been deleted by homologous recombination [24]. This experimental system provided a useful tool to study the role of p53 in the response of HCT116 cells to PTX. Nanomolar concentrations of the drug arrested HCT116 cells in G2/M before entering mitosis regardless of the presence of functional p53, which resulted in an altered distribution of cells in the cell cycle phases. In the presence of PTX, the percentage of polyploidy and of large multinucleate cells was higher in $p 53^{(-/)}$cells, indicating that mitotic slippage was more favored in p53-deficient than in wild-type cells. PTX caused mitotic catastrophe, and about 50\% cells entered aberrant mitosis and died through an apoptoticlike route. Apoptosis, which occurred in the absence of significant activation of caspases, was estimated by Annexin-V-Fluos cell staining due to the presence of phosphatidylserine in the outer 
membrane of apoptotic cells [25]. Even though the lack of p53 facilitated endoreduplication and polyploidy, cells that were able to overcome the checkpoints were killed by the same mechanism as wild-type cells after re-entering the cell cycle.

\section{Materials and methods}

\subsection{Cell culture and drug treatments}

Wild-type HCT116 human colon carcinoma cells and a p53 $3^{(-/-)}$derived cell line were kindly provided by Dr. B. Vogelstein (John Hopkins University, Baltimore). Cells were maintained in 50\% Dulbecco's MEM (Invitrogen)/50\% Ham's F12 (Lonza) medium, supplemented with 10\% fetal bovine serum (Invitrogen), $100 \mathrm{U} / \mathrm{ml}$ penicillin, $100 \mathrm{mg} / \mathrm{ml}$ streptomycin, at $37^{\circ} \mathrm{C}$ in a humidified atmosphere with 5\% $\mathrm{CO}_{2}$. A $1 \mathrm{mM}$ paclitaxel (Sigma) stock solution was prepared in DMSO, maintained at $-20^{\circ} \mathrm{C}$, and brought to the final concentration just before use.

Exponentially growing cells subcultured at a density of $2.5 \times 10^{4}$ cells $/ \mathrm{ml}$ were incubated with various concentrations of paclitaxel (PTX) at $37^{\circ} \mathrm{C}$. Moreover, $2 \mathrm{mM}$ caffeine (Sigma) was also added in some experiments (see section 3.3).

\subsection{Assessment of cell proliferation and viable cell number}

The effect of PTX on HCT116 cell growth was determined by using the MTT-method as described elsewhere [26]. In brief, cells subcultured at a density of $5 \times 10^{4}$ cells $/ \mathrm{ml}$ were incubated with various concentrations of PTX at $37^{\circ} \mathrm{C}$ for $72 \mathrm{~h}$. After incubation, 3-(4,5-dimethylthiazol-2yl)-2,5-diphenyltetrazolium (MTT, Sigma) was added to each culture ((50 mg)/well). The darkcolored crystals produced by viable cells were solubilized with $30 \mathrm{mM} \mathrm{HCl}$ in 2-propanol. Absorbance was determined at $570 \mathrm{~nm}$ using a BioTek ELx800 microplate reader.

To determine the capacity of PTX to produce cell death, the reduction in the number of viable cells was assessed at different time intervals by assessment of the exclusion of Trypan blue (Sigma), as described elsewhere [23].

In some experiments, co-treatments with the pan-caspase inhibitor Z-VAD-fmk (benzyloxycarbonyl-Val-Ala-Asp-fluoromethylketone) were used to evaluate the effects of caspase inactivation on cell death upon treatments with PTX. Cells were incubated for 24, 48 or $72 \mathrm{~h}$ with $7 \mu \mathrm{M}$ Z-VAD-fmk (Alexis) and $95 \mathrm{nM}$ PTX. As a positive control, cells were committed to dying by treatment with 55 nM WP631 (a gift of Prof. W. Priebe, MD Anderson Cancer Center, Houston), and $7 \mu \mathrm{M} Z$ Z-VAD-fmk used to inhibit caspase-dependent cell death induced by 48-h treatments with WP631.

\subsection{Analysis of cell cycle distribution and cell morphology after treatment}


After treatment with PTX for various periods of time, the cells were harvested, fixed with $70 \%$ ethanol and stained with PI (propidium iodide, Sigma) as described elsewhere [26]. Nuclei were analyzed with a Coulter Epics-XL flow cytometer, using the $488 \mathrm{~nm}$ line of an argon laser and standard optical emission filters.

Cells were morphologically analyzed before and after PTX treatments by phase-contrast microscopy, using a Nikon Eclipse TS100-F microscope.

2.4. Cytometric assessment of apoptotic and necrotic cell death

Apoptosis was determined by using the Annexin-V-Fluos staining kit (Roche Diagnostics) and flow cytometry in a Coulter Epics-XL flow cytometer. Apoptotic cells were characterized as two populations: Annexin-V-Fluos positive/PI negative (primary apoptotic cells) and Annexin-VFluos positive/PI positive (secondary apoptosis/necrosis).

\subsection{Changes in mitochondrial membrane potential}

To measure the changes in mitochondrial membrane potential $\left(\Psi_{\mathrm{mi}}\right)$, untreated and PTXtreated cells were stained with the MitoProbe DilIC(5) Assay kit (Molecular Probes) following the supplier's protocol and analyzed in a Beckman Coulter Gallios Cytometer.

\subsection{Determination of mitotic index and assessment of abnormal mitoses}

To analyze the mitotic fraction, control and PTX-treated cells were fixed and incubated with the anti-phospho-histone H3 (ser10) antibody (Millipore) followed by Cy2-conjugated secondary antibody (Jackson ImmunoResearch). Stained cells were counterstained with PI and analyzed for Cy2 and PI fluorescence, and quantified by flow cytometry.

For the analysis of mitotic figures, about 50-100 $\mu$ l of untreated or PTX-treated cells were dropped on clean slides with a Pasteur pipette and left to air-dry. Cells were fixed using Carnoy's solution (3 vol. methanol, 1 vol. glacial acetic acid). Slides were stained for 30 min with DAPI (Sigma) diluted in methanol, and examined under a Nikon E-800 fluorescence microscope.

\subsection{Western analysis of protein levels}

Protein was extracted from PTX-treated and untreated cells with a lysis buffer consisting of $50 \mathrm{mM}$ Tris- $\mathrm{HCl}$ (pH 8), $150 \mathrm{mM} \mathrm{NaCl}, 5 \mathrm{mM}$ EDTA, 0.5\% Nonidet P-40 and $0.1 \mathrm{mM}$ phenylmethylsulfonyl fluoride, containing $2 \mu \mathrm{g} / \mathrm{ml}$ aprotinin and $1 \mu \mathrm{g} / \mathrm{ml}$ leupeptin. Total protein was quantified by the Bradford assay (Bio-Rad). About $30 \mu \mathrm{g}$ of denatured proteins was subjected to electrophoresis on SDS-polyacrylamide gels, blotted onto Optitran BA-S85 membranes (Schleicher \& Schuell), probed with specific antibodies against $\mathrm{p} 21^{\mathrm{WAF} 1}$ (Calbiochem), Histone H3[pS10] (Millipore), p53, cyclin D and cyclin B1 (all from Santa Cruz), cleaved (active) caspase 3 (Cell Signaling Technology), active caspase 2 (AbCam), and GAPDH (Sigma), incubated with 
secondary antibodies (Jackson ImmunoResearch), and detected by chemiluminescence using Immobilon Western HRP Substrate (Millipore).

\section{Results}

Preliminary studies showed that low nanomolar concentrations of PTX produced a transient halt of wild-type HCT116 cells in G2/M phase, which was too short to allow us to examine them experimentally, while micromolar concentrations of the drug were very toxic producing too high cell death levels after short incubation times. From these preliminary experiments, we calculated $95 \mathrm{nM}$ PTX to be an ideal concentration to analyze the rise of mitotic catastrophe in wild-type HCT116 cells (details no reported).

\subsection{Nanomolar concentrations of PTX induced mitotic catastrophe in wild-type HCT116 human colon carcinoma cells}

Both cell proliferation and viability, measured by the MTT assay and Trypan blue exclusion respectively (see section 2.2.), indicated that the effects of $95 \mathrm{nM}$ PTX in wild-type HCT116 cells were time-dependent (Fig. 1A). After $72 \mathrm{~h}$, about $65 \%$ cells were viable although only around $15 \%$ were proliferating. Untreated cells remained in almost the same distribution in the different phases of the cell cycle (Fig. 1B, upper panels), while cells treated with PTX for $24 \mathrm{~h}$ accumulated in G2/M (Fig. 1B, lower panels). Both adherent (attached) cells and floating (detached) cells were analyzed separately. After 24-h treatments, the latter were mostly dead cells, as estimated by their staining with Trypan blue.

Morphological examination of cells that remained adherent upon treatment revealed the presence of some large multinucleate cells (Fig. 1C), indicating that some cells were entering a faulty mitosis without cytokinesis. After $24-\mathrm{h}$ treatments, we observed $43.6 \%$ mitotic cells, while only 3.8\% untreated cells were in mitosis (Fig. 1D). In PTX-treated wild-type HCT116 cells, mitotic figures showed a variety of abnormalities, including multipolar anaphases, uneven chromosome distribution and lagging telophases (Supplementary Fig. S1)

To further address the characteristics of the transient stop in G2/M in the presence of $95 \mathrm{nM}$ PTX, the levels of some protein involved in the control of G1 and G2/M checkpoints (p21 ${ }^{\text {WAF1, }}$ cyclin D1, cyclin B1) were analyzed by Western blot, as well as the presence of the mitotic form of Histone H3 (Histone H3[pS10]) (Fig. 1E). PTX induced the expression of p2 ${ }^{\text {WAF1 }}$ (Fig. 1E), while cyclin B1 levels were reduced in a time-dependent way, and this was concomitant with an enhancement of the cyclin D1 levels.

3.2. HCT116 cells treated with nanomolar concentrations of PTX were committed to dying by a caspase-independent route 
Neither caspase 2 nor caspase 3 were activated after treatment of wild-type HCT116 cells with 95 nM PTX (Fig. 1E). Because both caspases may be involved in the commitment of cells to mitotic catastrophe and apoptosis, we undertook additional experiments to explore whether cells entering mitosis, according to the presence of Histone H3[pS10] (Figs. 1D and E), could be dying by apoptosis or necrosis and whether other caspases may be implicated in the final fate of cells.

To discriminate between apoptotic and necrotic cell death, cells were co-stained with PI and Annexin-V-Fluos. Cells showing high levels of staining with Annexin-V-Fluos, which measures the presence of phosphatidylserine in the outer membrane of apoptotic cells, and low levels of PI were scored as apoptotic (Fig. 1F). Moreover, we also scored secondary apoptosis/necrosis characterized by having high levels of staining with both Annexin-V-Fluos and PI. As the percentage of primary necrosis was rather low after treatment with PTX (high staining with PI and low staining with Annexin-V-Fluos in Fig. 1F), we confidently scored the double-stained cells as being mainly secondary apoptosis. These experiments were performed in duplicate with similar results. Quantification of one of them is documented in the figure.

On the other hand, wild type HCT116 cells were treated with PTX in the presence of Z-VADfmk, a pan-inhibitor of caspases, thus no caspase could activate alternative caspase-dependent apoptotic pathways. To substantiate that the concentration of the pan-caspase inhibitor was enough to abrogate the caspase-dependent cell death routes, we used bis-anthracycline WP631 to induce caspase-dependent cell death. Nanomolar concentrations of WP631 induced cell death in HCT116 cells (Fig. 2A), an effect strongly inhibited by the presence of the pan-caspase inhibitor at the same concentrations used when examining PTX. The $y$-axis scales in the different panels in Fig. 2A were kept constant (i.e. a similar number of events were scored by cytometry in all the experiments). The different panels in Fig. 2A correspond to the living cells that remained attached, while those detached (floating) cells were mostly dead (not shown). The rather small number of cells seen in the '2nd panel' indicated the potent caspase-dependent apoptotic effect of WP631 in the absence of the pan-caspase inhibitor. Altogether, these results validated that the concentration of the inhibitor used in our experiments was enough to abrogate the response of caspases to cell damage, because only $6.8 \%$ cells died after treatment with WP631 in presence of the inhibitor (Fig. 2A, right panel). Figure 2B shows cell cycle distribution after treatments with $95 \mathrm{nM}$ PTX in the absence/presence of Z-VAD-fmk. In the presence of the inhibitor, PTX-treated cells were halted at the same checkpoints, though the number of floating cells decreased, and it was more evident the presence of polyploid ( $>4 \mathrm{~N}$ ) cells (cf. lower panels in Figs. 1B and 2B). Furthermore, the number of apoptotic and necrotic cells was not significantly altered by the presence of ZVAD-fmk (Fig. 2C), supporting the hypothesis of a caspase-independent mechanism of cell death 
upon mitotic catastrophe. Besides, the absence of significant changes in mitochondrial membrane potential after treatment with PTX (Fig. 2D) strengthens our idea that the intrinsic pathway was not involved in the mechanism of cell death.

\subsection{Caspase-independent cell death caused by PTX in HCT116 cells occurred regardless of the} p53 status

To elucidate whether the caspase-independent apoptotic cell death observed upon treatment with 95 nM PTX may be somehow dependent on p53, we took advantage of HCT116 cells whose p53 gene had been deleted by homologous recombination [24]. The time-dependent antiproliferative effect of $95 \mathrm{nM}$ PTX in HCT116 $p 53^{(-/-)}$cells is shown in Fig. 3A, together with a decrease in cell viability induced by the drug. Treatment of these cells with caffeine plus PTX, which made them to override the G2 checkpoint, had little effect on both proliferation and cell viability. Caffeine increased the percentage of polyploid cells after treatment (Fig. 3B). The presence of large multinucleate cells was documented using phase contrast microscopy (Fig. 3C). The mitotic index was quantified, with $56.5 \%$ p53-deficient HCT116 cells being in mitosis after 24-h treatments (Fig. 3D). PTX-treated p53-deficient HCT116 cells also showed a variety of mitotic abnormalities, including multipolar anaphases, uneven chromosome distribution and lagging telophases (Supplementary Fig. S1).

At this point, it is worth emphasizing that the major difference between the behavior of wildtype and p53-deficient HCT116 cells was the higher proportion of polyploid cells that would arise by endoreduplication in $\mathrm{p} 53^{--}$cells. Cells contained the mitotic Histone H3[pS10] after 24-h treatments (Fig. 3E), but they did not appear to be in mitosis afterwards. HCT116 $p 53^{(-/)}$deficient cells might undergo mitotic slippage more easily, re-entering a new cell cycle with altered ploidy, which occurred regardless of the presence/absence of caffeine. Furthermore, the absence of p $21^{\mathrm{WAF} 1}$ reactive to specific antibodies (Fig. 3E) implied that cells presented a weakened control on G1 and G2/M checkpoints. These results indicated that a failure to induce $\mathrm{p} 21^{\mathrm{WAF} 1}$ expression in p53-deficient cells resulted in the abrogation of the G2 checkpoint, consistent with previous observations [24,27]. Changes in the levels of cyclin D1 and cyclin B1 (Fig. 3E) were in keeping with the observed changes in cell cycle distribution shown in Fig. 3B.

To explore the presence of apoptosis and necrosis, drug-treated cells were co-stained with Annexin-V-Fluos and PI. Cells showing high levels of staining with Annexin-V-Fluos and low levels of PI were scored as primary apoptosis (Fig. 3F). Results of those quantifications are presented in the figure panels. It is noteworthy that the percentages of cell death measured in either wild-type or $p 53^{(-/-)}$cells were similar after $72-\mathrm{h}$ treatment (cf. Figs. 1F and 3F). 


\section{Discussion}

It has been suggested that mitotic catastrophe is not a genuine cell death executioner mechanism, but a pathway that precedes cell death or senescence $[12,13]$, and a large variety of in vitro and a few in vivo models have shown that it can shift to apoptosis or necrosis [1,3]. This shift appears to be a general principle and depends on the cell model examined, the drug used, and its dose [9-11,28]. After entering a faulty or aberrant mitosis cells can die during mitotic arrest or they can reach a new cell cycle round (mitotic slippage).

While apoptosis has been assumed to be the principal mechanism of cell death upon chemotherapeutic treatments, it has been suggested that in certain cancer cell lines PTX does not induce apoptosis but it can inhibit proliferation through other mechanisms depending on the cell type and the drug concentration [29,30]. In this context, p53 levels may depend on the PTX concentration used [31], so that low concentrations, like those used here, might reduce the functional transactivatory properties of $\mathrm{p} 53$, although this effect should be lower than that we observed in p53-deficient cells (cf. Figs. 1 and 3).

It is known that the induction of $\mathrm{p} 53$ and $\mathrm{p} 21^{\mathrm{WAF} 1}$ might produce either primary or postmitotic G1 arrest while inhibiting apoptosis, and that $\mathrm{p} 21^{\mathrm{WAF} 1}$ induction is fundamental for G1 arrest caused by microtubule dysfunction [30]. Here, we have shown that $\mathrm{p} 21^{\mathrm{WAF} 1}$ is induced after 72-h treatments of wild type HCT116 human colon carcinoma cells treated with nanomolar concentrations of PTX (Fig. 1E). Concomitantly, there was an increase in cyclin D1, in agreement with a halt at the G1-checkpoint of cells re-entering the cell cycle from mitotic slippage. This observation is consistent with the presence of next cell cycle with G1-like arrest. Besides, such a next cell cycle G1-like arrest can occur regardless of whether cells bear wild-type p53 (cf. Figs. $1 \mathrm{~B}$ and 3B and [19]). Figure 1B shows that G1-like cells were mostly detached (floating) dead cells, as they stained with Trypan blue. However, caspases were not significantly activated. This observation is at variance with the view that $\mathrm{p} 21^{\mathrm{WAF} 1}$ induction, which we already observed in wild-type cells (Fig. 1E) but not in p53-deficient ones (Fig. 3E), might cause post-mitotic arrest while inhibiting apoptosis. According to our results about 54\% p53-deficient cells might be dying, in absence of $\mathrm{p} 21^{\mathrm{WAF} 1}$ via an apoptotic-like route since they showed high Annexin-V-Fluos labeling.

The percentage of cell death in wild-type HCT116 cells was slightly changed by the presence of a pan-caspase inhibitor (Fig. 2B). The absence of significant activation of caspase 2 and caspase 3 can entail a caspase-independent apoptosis [13]. In any case, our results are consistent with that the therapeutic efficacy of taxanes would depend on whether slippage after mitotic arrest culminates in death by apoptosis or continued cell survival $[5,32]$. The experiments undertaken 
with $p 53^{(--)}$cells showed a slightly lower percentage of cell death (Fig. 3E). Besides, the clear increase in the percentage of polyploidization would indicate that cells that were not committed to dying by caspase-independent apoptosis after 72-h treatments were re-entering the cell cycle with endoreduplication and absence of cytokinesis. The percentage of polyploid cells increased from about $32 \%$ to $45 \%$ when caffeine was added. This suggests that in p53-deficient cells mitotic catastrophe produced a surviving cell population, in agreement with that endoreduplication may result from the abrogation of mitotic checkpoint control [28].

Our statement that about 50-60\% HCT116 cells died through an apoptotic-like route after 72h continuous treatment with nanomolar concentrations of PTX, even though neither caspase 3 nor caspase 2 active forms were detected, comes mainly from cell staining with Annexin-V-Fluos. This measures the presence of phospholipids, such as phosphatidylserine, in the outer membrane of cells undergoing apoptosis, but not of normal cells [25]. We have also examined PTX-treated cells in the presence of a pan-caspase inhibitor. This inhibitor did not alter the patterns of cell death significantly. However, it is conceivable that inhibition of caspases, other than caspases 2 and 3, could be insufficient and therefore low levels of other caspases may partially drive cell death. We also show that post-mitotic cell death is largely independent of p53 and is only partially suppressed by the apical caspase inhibitor Z-VAD-fmk. These findings suggest that progression through mitosis following DNA damage initiates a cell death response that is independent of p53 and caspases, which might prevent propagation of genetic lesions [33]. The presence of a higher percentage of polyploid $p 53^{(-/)}$cells after treatment was in line with that prevention of polyploidy requires a p53-dependent $\mathrm{G} 1$ checkpoint and a functional spindle checkpoint [8,34].

Caspase-independent cell death pathways are considered to be an important safeguard mechanisms to protect the organism against unwanted and potential harmful cells when caspasemediated routes fail but can also be triggered in response to cytotoxic agents or other death stimuli [35]. Here, we have demonstrated that these pathways are operative in HCT116 human colon carcinoma cells upon PTX treatment, in keeping with the view that they may depend in drug doses and cell types [9-11]. Although it has been previously reported that mitotic catastrophe can result in cell death by caspase-independent mechanisms (reviewed in [15]), our results peculiarly show cells that were killed by apoptosis rather than by necrosis. In the absence of direct evidence about the mechanisms and molecules involved in the apoptotic cell death that is working under our experimental conditions, it is clear that alternative protease activities must be capable of inducing apoptotic-like death [36]. Tentatively, we consider that some cathepsins, which possess strong proteolytic activities and have linked to apoptosis [36,37], could be operative after PTX treatment. 
We have observed that apoptotic-like cell death arises from mitotic catastrophe in both wildtype and $p 53^{(-/-)} \mathrm{HCT} 116$ cells. Induction of apoptosis may require both activation of the spindle assembly checkpoint and mitotic slippage [38]. Furthermore, some of the polyploid cells that were multinucleated and presented micronuclei (Figs. 1C and 3C) might still be eliminated by apoptosis executed by a Bax-dependent mitochondrial pathway [8], which can occur by p53 transcriptiondependent and -independent pathways [39]. Although the relation between the spindle checkpoint and cell death remains obscure, it has been suggested that BUB1 mediation of caspaseindependent mitotic death determines cell fate [40]. Furthermore, the perturbation of microtubule dynamics induces BIM translocation from microtubule to mitochondria, where it could exert its pro-apoptotic activity [41]. The absence of significant changes in the mitochondrial membrane potential after treatment with $95 \mathrm{nM}$ PTX (Fig. 2D) suggests that those pro-apoptotic responses would occur without important changes in mitochondrial physiology.

Given that p53 is known to be inactivated in most human cancers, mitotic catastrophe underlines the killing of cells after chemotherapy. At present, understanding how cells actually die during caspase-independent cell death is limited [42]. Further clarification of caspaseindependent cell death induced by anticancer drugs might lead to significant improvement of current chemotherapy.

\section{Conflict of interest}

None declared

\section{Acknowledgements}

This work was supported by grant BFU2010-15518 from the Spanish Ministry of Science and Innovation, and the FEDER program of the European Community, and it was performed within the framework of the "Xarxa de Referencia en Biotecnologia" of the Generalitat de Catalunya. 


\section{REFERENCES}

[1] I.B. Roninson, E.V. Broude, B.-D. Chang, If not apoptosis, then what? Treatment-induced senescence and mitotic catastrophe in tumor cells. Drug Resist. Updat. 4 (2001) 303-313.

[2] F. Ianzini, M. Mackey, Mitotic Catastrophe, in: D.A. Gewirtz, S.E. Holt, S. Grant, (Eds.), Apoptosis, Senescence and Cancer, Humana Press, New Jersey, 2007, pp. 73-91.

[3] S. Mansilla, M. Bataller, J. Portugal, Mitotic catastrophe as a consequence of chemotherapy. Anticancer Agents Med. Chem. 6 (2006) 589-602.

[4] M. Castedo, J.L. Perfettini, T. Roumier, K. Andreau, R. Medema, G. Kroemer, Cell death by mitotic catastrophe: a molecular definition. Oncogene 23 (2004) 2825-2837.

[5] K.E. Gascoigne, S.S. Taylor, How do anti-mitotic drugs kill cancer cells? J. Cell Sci. 122 (2009) 2579-2585

[6] S. Mansilla, L. Llovera, J. Portugal, Chemotherapeutic Targeting of Cell Death Pathways. Anticancer Agents Med. Chem. 12 (2012) 226-238.

[7] M. Bataller, C. Méndez, J.A. Salas, J. Portugal, Mithramycin SK modulates polyploidy and cell death in colon carcinoma cells. Mol. Cancer Ther. 7 (2008) 2988-2997.

[8] M. Castedo, A. Coquelle, S. Vivet, I. Vitale, A. Kauffmann, P. Dessen, M.O. Pequignot, N. Casares, A. Valent, S. Mouhamad, E. Schmitt, N. Modjtahedi, W. Vainchenker, L. Zitvogel, V. Lazar, C. Garrido, G. Kroemer, Apoptosis regulation in tetraploid cancer cells. EMBO J. 25 (2006) 2584-2595.

[9] S. Mansilla, W. Priebe, J. Portugal, Mitotic catastrophe results in cell death by caspasedependent and caspase-independent mechanisms. Cell Cycle 5 (2006) 53-60.

[10] Y.W. Eom, M.A. Kim, S.S. Park, M.J. Goo, H.J. Kwon, S. Sohn, W.H. Kim, G. Yoon, K.S. Choi, Two distinct modes of cell death induced by doxorubicin: apoptosis and cell death through mitotic catastrophe accompanied by senescence-like phenotype. Oncogene 24 (2005) 4765-4777.

[11] H. Vakifahmetoglu, M. Olsson, C. Tamm, N. Heidari, S. Orrenius, B. Zhivotovsky, DNA damage induces two distinct modes of cell death in ovarian carcinomas. Cell Death Differ. 15 (2008) 555-566.

[12] I. Vitale, L. Galluzzi, M. Castedo, G. Kroemer, Mitotic catastrophe: a mechanism for avoiding genomic instability. Nat. Rev. Mol. Cell Biol. 12 (2011) 385-392.

[13] L. Galluzzi, I. Vitale, J.M. Abrams, E.S. Alnemri, E.H. Baehrecke, M.V. Blagosklonny, T.M. Dawson, V.L. Dawson, W.S. El-Deiry, S. Fulda, E. Gottlieb, D.R. Green, M.O. Hengartner, O. Kepp, R.A. Knight, S. Kumar, S.A. Lipton, X. Lu, F. Madeo, W. Malorni, P. Mehlen, G. Nunez, M.E. Peter, M. Piacentini, D.C. Rubinsztein, Y. Shi, H.U. Simon, P. 
Vandenabeele, E. White, J. Yuan, B. Zhivotovsky, G. Melino, G. Kroemer, Molecular definitions of cell death subroutines: recommendations of the Nomenclature Committee on Cell Death 2012. Cell Death Differ. 19 (2012) 107-120.

[14] M. Abend, Reasons to reconsider the significance of apoptosis for cancer therapy. Int. J. Radiat. Biol. 79 (2003) 927-941.

[15] J. Portugal, S. Mansilla, M. Bataller, Mechanisms of drug-induced mitotic catastrophe in cancer cells. Curr. Pharm. Des. 16 (2010) 69-78.

[16] M. Bataller, J. Portugal, Apoptosis and cell recovery in response to oxidative stress in p53deficient prostate carcinoma cells. Arch. Biochem. Biophys. 437 (2005) 151-158.

[17] B. Zhivotovsky, S. Orrenius, Cell death mechanisms: cross-talk and role in disease. Exp. Cell Res. 316 (2010) 1374-1383.

[18] M.A. Jordan, L. Wilson, Microtubules as a target for anticancer drugs. Nat. Rev. Cancer 4 (2004) 253-265.

[19] Z.N. Demidenko, S. Kalurupalle, C. Hanko, C.U. Lim, E. Broude, M.V. Blagosklonny, Mechanism of G1-like arrest by low concentrations of paclitaxel: next cell cycle p53dependent arrest with sub G1 DNA content mediated by prolonged mitosis. Oncogene 27 (2008) 4402-4410.

[20] S. Giovinazzi, C.R. Lindsay, V.M. Morozov, E. Escobar-Cabrera, M.K. Summers, H.S. Han, L.P. McIntosh, A.M. Ishov, Regulation of mitosis and taxane response by Daxx and Rassf1. Oncogene. 31 (2012) 13-26.

[21] G. Tan, L. Heqing, C. Jiangbo, J. Ming, M. Yanhong, L. Xianghe, S. Hong, G. Li, Apoptosis induced by low-dose paclitaxel is associated with p53 upregulation in nasopharyngeal carcinoma cells. Int J Cancer. 97 (2002) 168-172.

[22] M.E. Bekier, R. Fischbach, J. Lee, W.R. Taylor, Length of mitotic arrest induced by microtubule-stabilizing drugs determines cell death after mitotic exit. Mol Cancer Ther. 8 (2009) 1646-1654.

[23] M. Bataller, C. Méndez, J.A. Salas, J. Portugal, Cellular response and activation of apoptosis by mithramycin SK in p2 $1^{\mathrm{WAF} 1}$-deficient HCT116 human colon carcinoma cells. Cancer Lett. 292 (2010) 80-90.

[24] F. Bunz, A. Dutriaux, C. Lengauer, T. Waldman, S. Zhou, J.P. Brown, J.M. Sedivy, K.W. Kinzler, B. Vogelstein, Requirement for p53 and p21 to sustain G2 arrest after DNA damage. Science 282 (1998) 1497-1501.

[25] Z. Darzynkiewicz, E. Bedner, P. Smolewski, Flow cytometry in analysis of cell cycle and apoptosis. Semin. Hematol. 38 (2001) 179-193. 
[26] S. Villamarín, S. Mansilla, N. Ferrer-Miralles, W. Priebe, J. Portugal, A comparative analysis of the time-dependent antiproliferative effects of daunorubicin and WP631. Eur. J. Biochem. 270 (2003) 764-770.

[27] M. Nitta, O. Kobayashi, S. Honda, T. Hirota, S. Kuninaka, T. Marumoto, Y. Ushio, H. Saya, Spindle checkpoint function is required for mitotic catastrophe induced by DNAdamaging agents. Oncogene 23 (2004) 6548-6558.

[28] B.D. Chang, E.V. Broude, J. Fang, T.V. Kalinichenko, R. Abdryashitov, J.C. Poole, I.B. Roninson, $\mathrm{p} 21^{\mathrm{Waf1} / \mathrm{Cip} 1 / \mathrm{Sdi1}}$-induced growth arrest is associated with depletion of mitosiscontrol proteins and leads to abnormal mitosis and endoreduplication in recovering cells. Oncogene 19 (2000) 2165-2170.

[29] C. Lanzi, G. Cassinelli, G. Cuccuru, R. Supino, V. Zuco, C. Ferlini, G. Scambia, F. Zunino, Cell cycle checkpoint efficiency and cellular response to paclitaxel in prostate cancer cells. Prostate. 48 (2001) 254-264.

[30] M.V. Blagosklonny, Z. Darzynkiewicz, H.D. Halicka, P. Pozarowski, Z.N. Demidenko, J.J. Barry, K.R. Kamath, R.A. Herrmann, Paclitaxel induces primary and postmitotic G1 arrest in human arterial smooth muscle cells. Cell Cycle 3 (2004) 1050-1056.

[31] M.L. Panno, F. Giordano, F. Mastroianni, C. Morelli, E. Brunelli, M.G. Palma, M. Pellegrino, S. Aquila, A. Miglietta, L. Mauro, D. Bonofiglio, S. Ando, Evidence that low doses of Taxol enhance the functional transactivatory properties of p53 on p21 waf promoter in MCF-7 breast cancer cells. FEBS Lett. 580 (2006) 2371-2380.

[32] P. Russell, B.T. Hennessy, J. Li, M.S. Carey, R.C. Bast, T. Freeman, A.R. Venkitaraman, Cyclin G1 regulates the outcome of taxane-induced mitotic checkpoint arrest. Oncogene 31 (2012) 2450-2460.

[33] H. Varmark, C.A. Sparks, J.J. Nordberg, B.S. Koppetsch, W.E. Theurkauf, DNA damageinduced cell death is enhanced by progression through mitosis. Cell Cycle 8 (2009) 29512963.

[34] C. Vogel, A. Kienitz, I. Hofmann, R. Muller, H. Bastians, Crosstalk of the mitotic spindle assembly checkpoint with p53 to prevent polyploidy. Oncogene 23 (2004) 6845-6853.

[35] L.E. Broker, F.A. Kruyt, G. Giaccone, Cell death independent of caspases: a review. Clin. Cancer Res. 11 (2005) 3155-3162.

[36] L. Foghsgaard, D. Wissing, D. Mauch, U. Lademann, L. Bastholm, M. Boes, F. Elling, M. Leist, M. Jaattela, Cathepsin B acts as a dominant execution protease in tumor cell apoptosis induced by tumor necrosis factor. J Cell Biol. 153 (2001) 999-1010. 
[37] P. Kreuzaler, C.J. Watson, Killing a cancer: what are the alternatives? Nat Rev Cancer 12 (2012) 411-424.

[38] W. Tao, V.J. South, Y. Zhang, J.P. Davide, L. Farrell, N.E. Kohl, L. Sepp-Lorenzino, R.B. Lobell, Induction of apoptosis by an inhibitor of the mitotic kinesin KSP requires both activation of the spindle assembly checkpoint and mitotic slippage. Cancer Cell 8 (2005) 49-59.

[39] H. Yamaguchi, J. Chen, K. Bhalla, H.G. Wang, Regulation of Bax activation and apoptotic response to microtubule-damaging agents by p53 transcription-dependent and independent pathways. J. Biol. Chem. 279 (2004) 39431-39437.

[40] Y. Niikura, A. Dixit, R. Scott, G. Perkins, K. Kitagawa, BUB1 mediation of caspaseindependent mitotic death determines cell fate. J. Cell Biol. 178 (2007) 283-296.

[41] O. Kutuk, A. Letai, Displacement of Bim by Bmf and Puma rather than increase in Bim level mediates paclitaxel-induced apoptosis in breast cancer cells. Cell Death Differ. 17 (2010) 1624-1635.

[42] S.W. Tait, D.R. Green, Caspase-independent cell death: leaving the set without the final cut. Oncogene 27 (2008) 6452-6461. 


\section{LEGENDS TO FIGURES}

Fig. 1. (A) Time-dependent effects of $95 \mathrm{nM}$ PTX on cell proliferation and viability of wild-type HCT116 human colon carcinoma cells. Data are means \pm SD for three independent experiments. (B) Cell cycle distribution of HCT116 cells treated continuously with 95 nM PTX. Black lines correspond to adherent cells, while gray lines correspond to detached (floating) cells, which were mostly dead. (C) Phase-contrast microscopy of untreated HCT116 cells (left panel) and cells treated with $95 \mathrm{nM}$ PTX for $48 \mathrm{~h}$ showing the presence of large multinucleate cells (right panel). These cells were a minority and thus not easy to quantify in panel B (cf. with the same panel in Fig. 3). Bars correspond to $50 \mu \mathrm{m}$. (D) Assay for the detection of cells in mitosis by an antibody against H3Sp10 (mitotic phosphorylated form of histone H3). The percentage of mitotic cells (mitotic index) is indicated. (E) Western blots showing changes in the levels of several proteins in wild-type HCT116 cells treated with 95 nM PTX for the times indicated at the top of the panel. The active forms of caspase 2 and caspase 3 were not detected in both untreated and drug-treated cells. GAPDH was used as normalization control. (F) Flow cytometry of wild type HCT116 cells co-stained with Annexin-V-Fluos and PI. Experiments were carried out in duplicate with similar results. The percentage of apoptotic cells is indicated in the plots. Both primary apoptotic cells (Annexin-V-Fluos positive/PI negative) and secondary apoptosis/necrosis (Annexin-V-Fluos positive/PI positive) cells populations were quantified.

Fig. 2. (A) A $7 \mu \mathrm{M}$ concentration of the pan-caspase inhibitor Z-VAD-fmk abrogates cell death induced by 55nM WP631 in HCT116 cells. This positive control shows that this concentration of Z-VAD-fmk was enough to inhibit apoptosis by about $95 \%$ - the different panels, which refer to cells adherent cells, are represented at the same scale to substantiate that WP631 induced cell death in the absence of the inhibitor but not in its presence-- (B) Effect of the Z-VAD-fmk in cell cycle distribution of wild-type HCT116 cells treated with 95 nM PTX. Numbers inside the panels indicate the percentage of polyploid cells, which increased in the presence of the inhibitor. (C) Flow cytometry of wild-type HCT116 cells co-stained with Annexin-V-Fluos and PI. Experiments were performed in duplicate with similar results, and the percentage of apoptotic cells is indicated in the plots. (D) Direct analysis of changes in mitochondrial membrane potential showing that treatment of wild-type HCT-116 cells with $95 \mathrm{nM}$ PTX does not produce a significant disruption of the membrane potential regardless of the presence/absence of the pancaspase inhibitor Z-VAD-fmk. Values are means \pm SD for three independent experiments. 
Fig. 3. (A) Time-dependent effects of $95 \mathrm{nM}$ PTX on cell proliferation and viability of HCT116 $p 53^{(-/-)}$human colon carcinoma cells in the pesence/absence of caffeine (CAF). Data are means \pm SD for three independent experiments. (B) Cell cycle distribution of HCT116 $p 53^{(-/)}$cells treated continuously with $95 \mathrm{nM}$ PTX. Black lines correspond to adherent cells, while gray lines correspond to detached (floating) cells, which were mostly dead. Numbers inside the panels are the percentage of polyploid ( $>4 \mathrm{~N}$ ) cells (CAF: caffeine). (C) Phase-contrast microscopy of untreated HCT116 cells (left panel) and cells treated with $95 \mathrm{nM}$ PTX for 24 h showing the presence of large multinucleate cells (right panel). These large cells were about $11 \%$ of the cell population upon treatments (see panel B). (D) Assay for the detection of cells in mitosis by an antibody against H3Sp10 (mitotic phosphorylated form of histone H3). The percentage of mitotic cells (mitotic index) is indicated. (E) Western blots showing changes in the levels of several proteins in HCT116 (p53 $3^{-/}$) cells treated with 95 nM PTX for the times indicated at the top of the panel. (F) Flow cytometry of HCT116 (p53/-) cells stained with Annexin-V-Fluos and PI. Experiments were carried out in duplicate with similar results. Other details as in legend to Fig. $1 \mathrm{~F}$. 
A

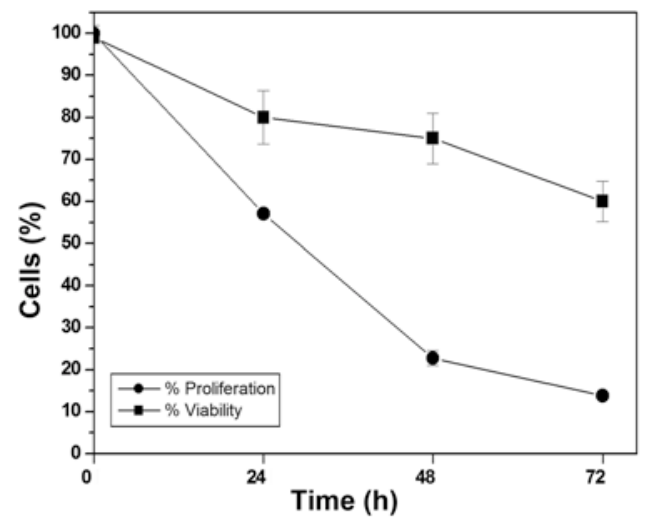

C
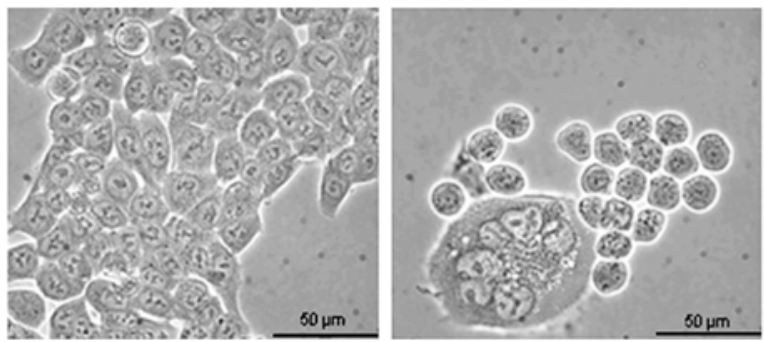

E

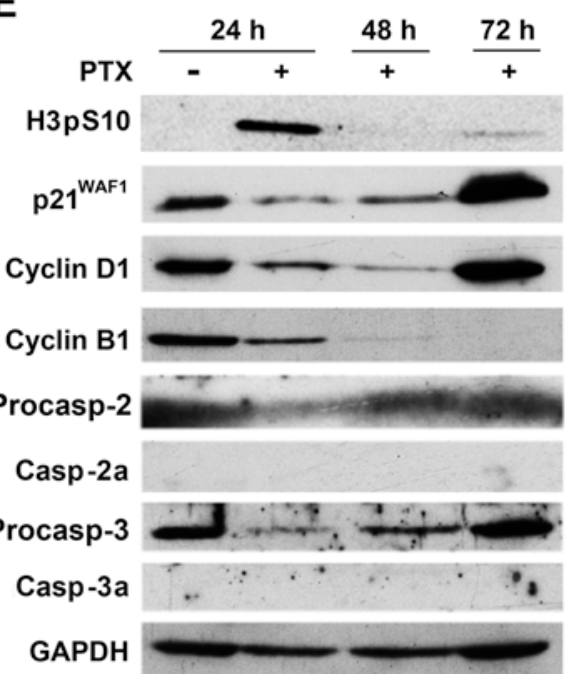

B

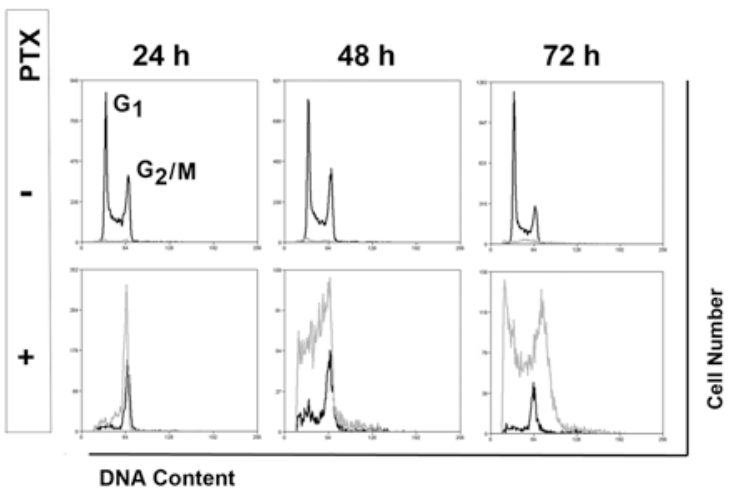

D

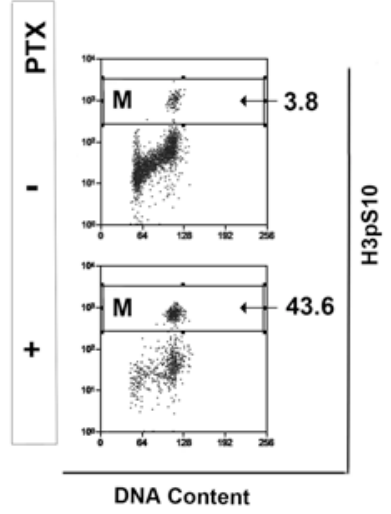

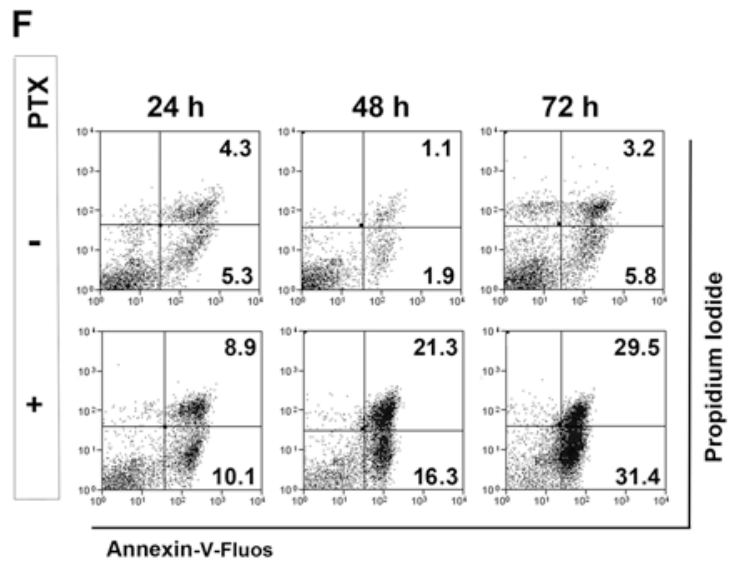

FIG. 1 
A

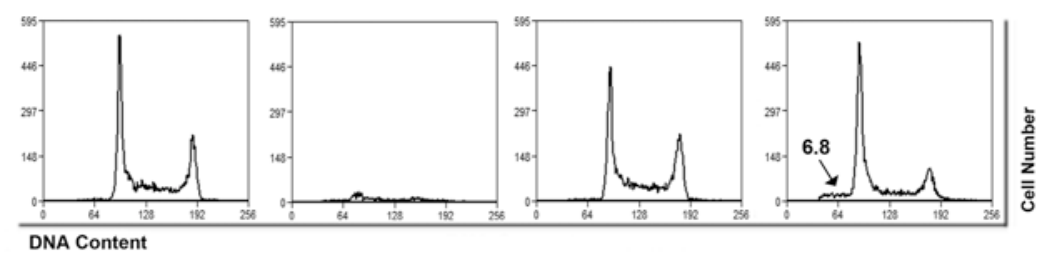

WP63
Z-VAD-FMK

B

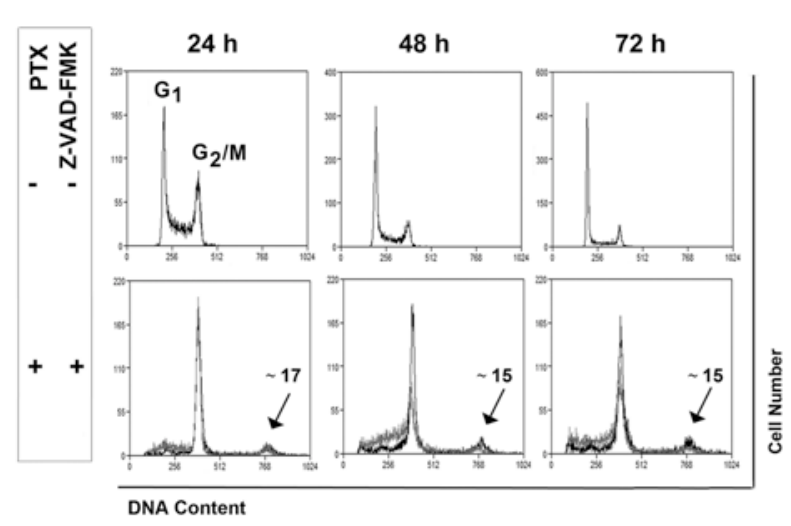

C

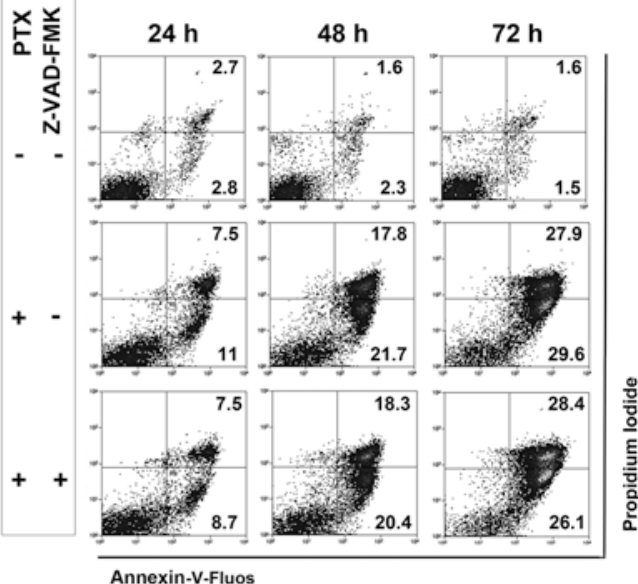

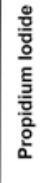

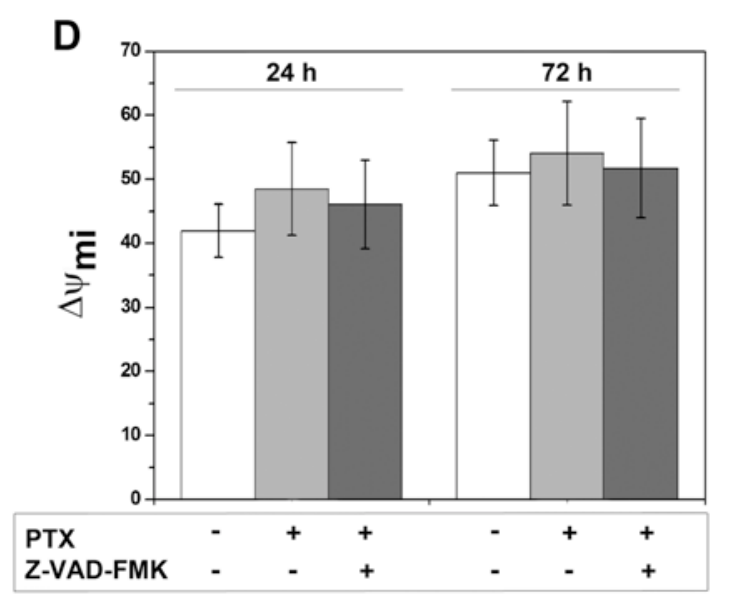

FIG. 2 
A

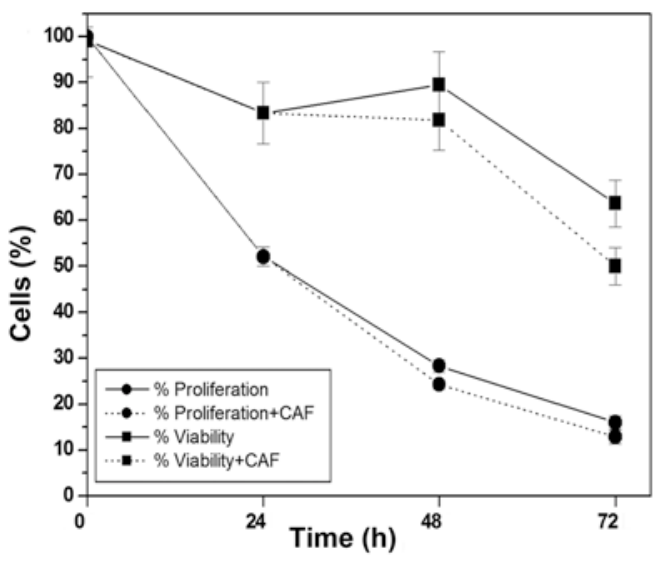

C
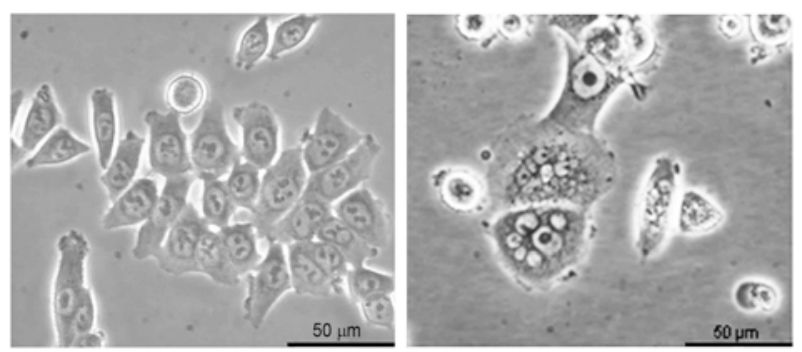

E
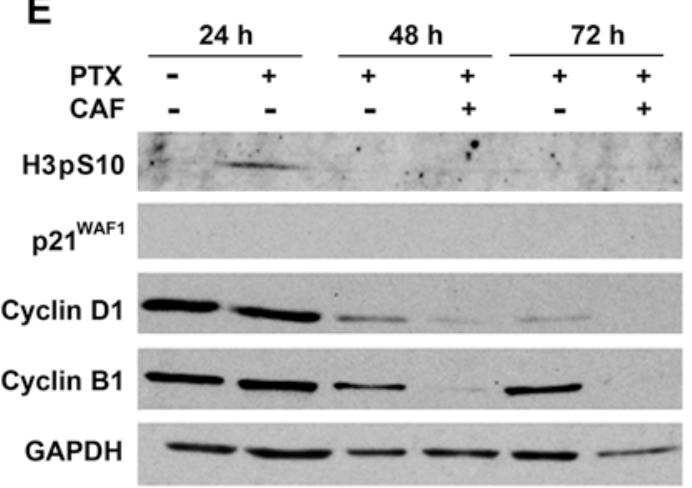

FIG. 3
B

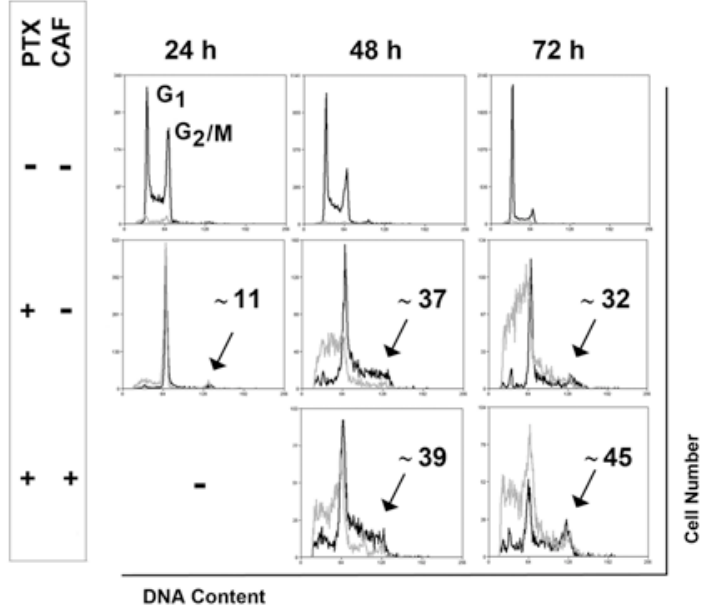

D

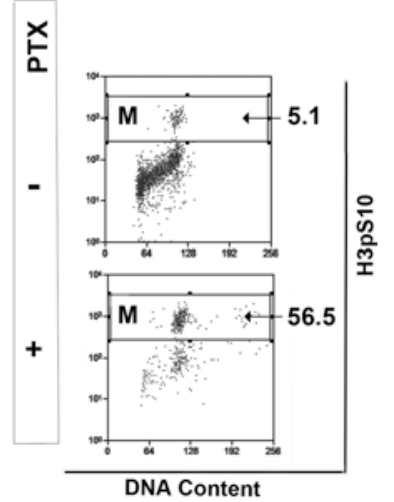

F

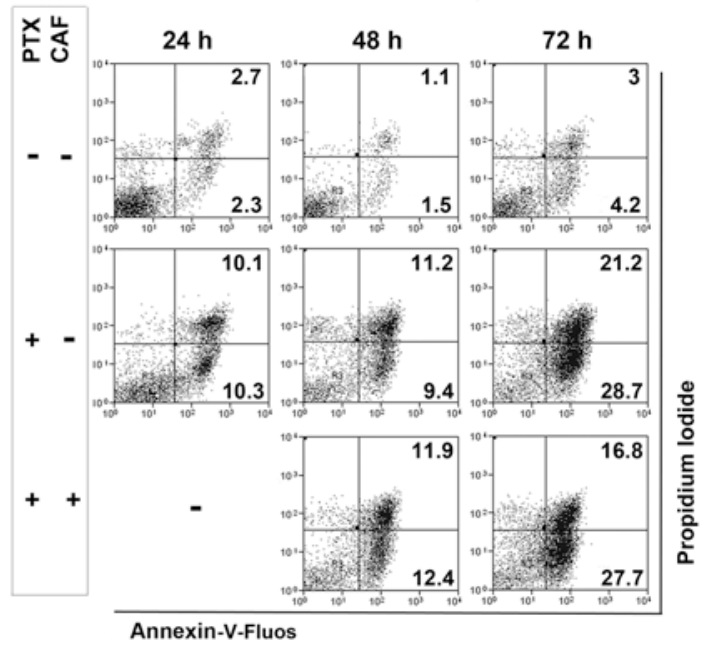

